\title{
A Seamless Mobility Scenario for IP over WiMAX
}

\author{
Vitor Bernardo, Luis Cordeiro, Isidro Caramelo, Bruno Sousa, Marilia Curado, \\ Edmundo Monteiro \\ Laboratory of Communications and Telematics, \\ Department of Informatics Engineering, University of Coimbra \\ CISUC - DEI, Polo II, 3030-290 Coimbra - Portugal \\ \{vmbern, cordeiro, isidro, bmsousa, marilia, edmundo\}@dei.uc.pt
}

\begin{abstract}
WiMAX is a broadband wireless access technology facing the challenges of $3 \mathrm{G}$ and beyond communication systems. As frequency licensing is becoming commercially available throughout the world, it is still unclear whether WiMAX will be better positioned than its main competitors, such as UMTS. Therefore, it is critical to evaluate the technology within the foreseen all-IP communications. In the context of the WEIRD project, an architecture that includes the WiMAX technology in an all-IP communication system has been developed. The main capabilities of the WEIRD system include mechanisms to support adequate quality of service levels for a broad range of applications, from basic web traffic to multimedia and specialized applications, such as telemedicine and fire prevention. This paper presents a solution for macro mobility in an all-IP network with WiMAX as the access technology, based on the basic WEIRD architecture. The proposed approach has been implemented on a test-bed of the WEIRD system and evaluated in order to address the impact of the developed mechanisms upon the overall system performance. The results have shown that the mobility mechanisms introduce a rather small overhead, while having the capability to support a "make-beforebreak" handover process.
\end{abstract}

Keywords: Mobility, QoS, WiMAX, NSIS, MIH

\section{$1 \quad$ Introduction}

Worldwide Interoperability for Microwave Access (WiMAX) has become a key technology for broadband wireless access in next generation networks, both in its fixed and mobile versions.

The WiMAX Extensions to Isolated Research Data networks (WEIRD) was a 24 month integrated project that aimed at implementing research test-beds via the usage of the WiMAX technology [1]. In WEIRD, the WiMAX technology is integrated in an all-IP network, which takes into account features such as Quality of Service (QoS), resource and access management, authentication authorization and accounting (AAA), and mobility [2]. Moreover, the WEIRD system supports a wide range of applications, from traditional web browsing, through Voice over IP, up to more 
elaborated applications, such as fire prevention and telemedicine. Currently, the fire prevention application is being used by the Portuguese Civil Protection.

The mobile version of WiMAX has native support for micro mobility. However it does not support, by itself, layer 3 mobility mechanisms, which need to be included in order to handle macro mobility. To overcome the limitations expressed above, this paper specifies and describes the preliminary evaluation of a solution for macromobility within the WEIRD project, following a "make-before-break" handover procedure. The most important modules involved in the macro-mobility scenario are the QoS modules for resource reservation both on the WiMAX links and on the core network, and the mobility management modules, including the Media Independent Handover. Moreover, an extension to the Next Steps in Signalling Framework for mobility management support was designed and integrated in the previously mentioned mobility scenario.

The paper is organized as follows: Section 2 describes the WiMAX technology, the signalling framework and the mobility handover assistance mechanism used in the WEIRD system. The related work is presented in the Section 3. Section 4 presents a detailed description of the proposed macro-mobility approach. The results of the proposal evaluation on the test-bed are discussed in Section 5. Finally, Section 6 concludes the paper and presents issues to be addressed in future work.

\section{WiMAX in all-IP: Quality of Service and Mobility}

An architecture that integrates the WiMAX technology in an all-IP world with QoS and mobility support comprises several components with specific roles. This section starts with a brief overview of the WiMAX technology, followed by the description of the signalling framework used in the WEIRD project. The modules involved in mobility support, namely the Mobile IP and the Media Independent Handover complete this section.

\subsection{WiMAX}

WiMAX, Worldwide Interoperability for Microwave Access, is a technology based on the IEEE 802.16 standard that aims to provide broadband wireless access over long distances, both fixed and mobile, as defined by the IEEE 802.16-2004 [3] and IEEE 802.16e-2005 [4] standards, respectively. The WiMAX Forum is the organization responsible for the certification and interoperability of all the products based on the IEEE 802.16 /ETSI HiperMAN standard [5][6]. The Network Working Group of WiMAX Forum has defined the WiMAX network reference model [7], shown in Fig. 1. 


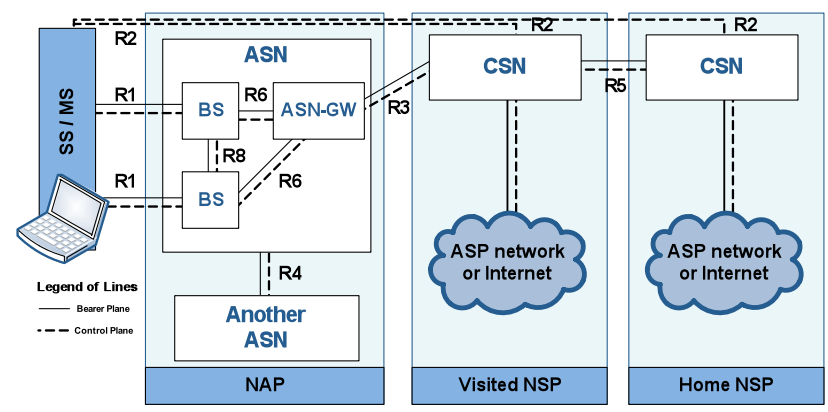

Fig. 1. WiMAX Forum Network Reference Model [7]

The Network Access Provider (NAP) is the entity that provides WiMAX radio access to the Network Service Providers (NSP) and controls the Access Service Network (ASN). The IP connectivity and the WiMAX services are provided by the NSP, which also manages the Connectivity Service Network (CSN). The communication between the different entities is performed through the following reference points:

- R1 - Protocols and procedures between the Mobile Station (MS) and the ASN;

- R2 - Protocols and procedures between the MS and CSN associated with authentication, services authorization and IP host configuration management;

- R3 - Control plane protocols between ASN and CSN to support AAA, policy enforcement and mobility;

- R4 - Control and bearer planes protocols between ASN-GWs to coordinate the MS mobility; interoperability between ASNs;

- R5 - Control and bearer planes protocols for internetworking between the CSN operated by home NSP and that operated by a visited NSP;

- R6 - Control and bearer planes protocols for communication between BS and ASN-GW;

- R7 - Optional reference point, which defines the control plane procedures between the Policy Decision Point (PDP) and the Policy Enforcement Point (PEP);

- R8 - Reference point defined by the IEEE 802.16e and IEEE 802.16g to enable the seamless handover. It specifies the control plane messages and bearer plane data flows between different BSs.

The WiMAX architecture supports two types of mobility: the ASN anchored mobility, also known as micro-mobility or intra-ASN mobility, and the CSN anchored mobility. The later, also known as macro-mobility or inter-ASN mobility, is IP based mobility between the ASN and CSN across the R3 reference point. This paper describes a macro-mobility scenario using WiMAX within the context of the WEIRD project.

\subsection{Next Steps in Signalling}

The Next Steps in Signalling (NSIS) [9] is a new framework that is being defined at the Internet Engineering Task Force (IETF). The NSIS framework is composed by 
two layers: NSIS Transport Layer Protocol (NTLP) that is a generic layer and a NSIS Signalling Layer Protocol (NSLP) that is specific to each application, as depicted in Fig. 2. For instance, the QoS-NSLP is responsible for resource reservation [12].

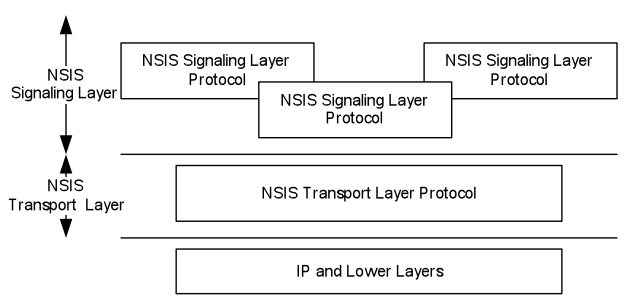

Fig. 2. NSIS framework structure

The NTLP layer, also named General Internet Signalling Transport (GIST) [11], is a lower layer responsible for the communication between all the peers in the network, by transporting the signalling messages sent by NSLPs. In the context of the WEIRD project, a new NSLP was proposed, the Media Independent Handover Network Signalling Layer Protocol (MIH-NSLP) [13]. The MIH-NSLP is used to transport the IEEE 802.21 [8] messages between MIH Function entities.

\subsection{Media Independent Handover}

The Media Independent Handover (MIH), identified as IEEE 802.21, is a proposed standard to support the seamless handover between heterogeneous technologies such as GSM, IEEE 802.11 and IEEE 802.16. The MIH standard defines a new entity, the Media Independent Handover Function (MIHF), which must be supported by all the elements involved in the handover operations. The MIHF is used as a channel to exchange information between the lower and upper layers, as shown in Fig. 3.

The MIH specification supports two different handover methods, namely, "breakbefore-make" or "hard" handover, and "make-before-break" or "soft" handover.

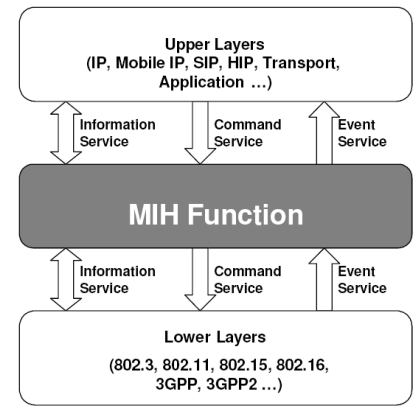

Fig. 3. MIH architecture [8]

The MIH standard specifies a Media Independent Information Service (MIIS), which provides detailed information about the neighbouring networks. This information is essential to support handover decisions in macro-mobility scenarios. The MIH standard and its capability to support a seamless service provision in 
heterogeneous networks is discussed in [19]. In the WEIRD architecture, MIH is employed to handle the events triggered by lower layers and which assist the handover procedures at upper layers.

\section{Related Work}

An architecture for mobility management between 3G and WiMAX networks is presented in [15]. This architecture is based on MIP to enable IP mobility and SIP to enable session mobility, therefore introducing support for the IMS architecture. Despite the interworking between different technologies, cross-layer information is not employed to improve the handover performance. Instead the architecture relies on layer 3 protocols to enable heterogeneous handovers.

Roland et al. [16] introduce an architecture for QoS signalling for IP mobile networks, combining resource management with mobility management procedures, resorting to reservation before performing the handover. Despite the support of anticipated handover and technology independence, the detection of new access points is solely based on layer 3 data. By employing cross-layer information, namely $\mathrm{MIH}$ facilities, the handover could be reduced and QoS signalling could be anticipated.

An integrated QoS-aware mobility architecture for seamless handover in IEEE $802.16 \mathrm{e}$ is presented in [17], where a new cross-layer mobility scheme has been developed with the help of the lower layers. Nevertheless, there is not any mechanism to enable the handover notification from the upper layers, for instance, by an entity responsible for the mobility management.

Dutta et al. [18] present an architecture for seamless handovers across heterogeneous access networks. This architecture is based on the facilities of MIH and the Media Independent Pre-Authentication (MPA) framework. This last one allows performing authentication at layer 2 and layer 3, independently of the technology. Besides introducing the design of the architecture, it is also evaluated in a test-bed based on the IEEE 802.11 and CDMA technologies. Despite the improved handover performance with the assistance of MIH and MPA, the transport of MIH messages between MIHF entities is based on the HTTP protocol, which introduces delay in event propagation, due to its connection-oriented nature. Moreover, the MIH standard does not require assured delivery, or other complex mechanisms present in this type of transport protocol.

\section{Mobility Scenario Description}

This section describes a WiMAX macro-mobility scenario following a "make-beforebreak" handover approach within the context of the WEIRD system. 
Edmundo Monteiro

\subsection{Motivation}

The mobility mechanisms needed to support full mobility with resource reservation over the WiMAX technology are the focus of this work. The mobility scenario described demonstrates the application of the Inter NAP R3 mobility, as defined by the WiMAX Forum. Given the immature state of the mobile WiMAX equipment and the associated high costs, the proposed mobility model is evaluated with IEEE 802.16d equipment. Therefore, the handover process will occur between IEEE 802.11 access points, which in turn are connected to a respective SS. This pragmatic approach does not prevent the proof of concept central to the proposal, since the upper layer mechanisms are oblivious to the lower layers, due to the use of the Media Independent Handover mechanisms.

\subsection{Make-before-break Handover Process}

The handover procedure of a Mobile Node (MN) between two ASNs is split into three different phases, namely, the triggering, provisioning and decision phases. Fig. 4 shows a simplified diagram of the proposed handover procedure.

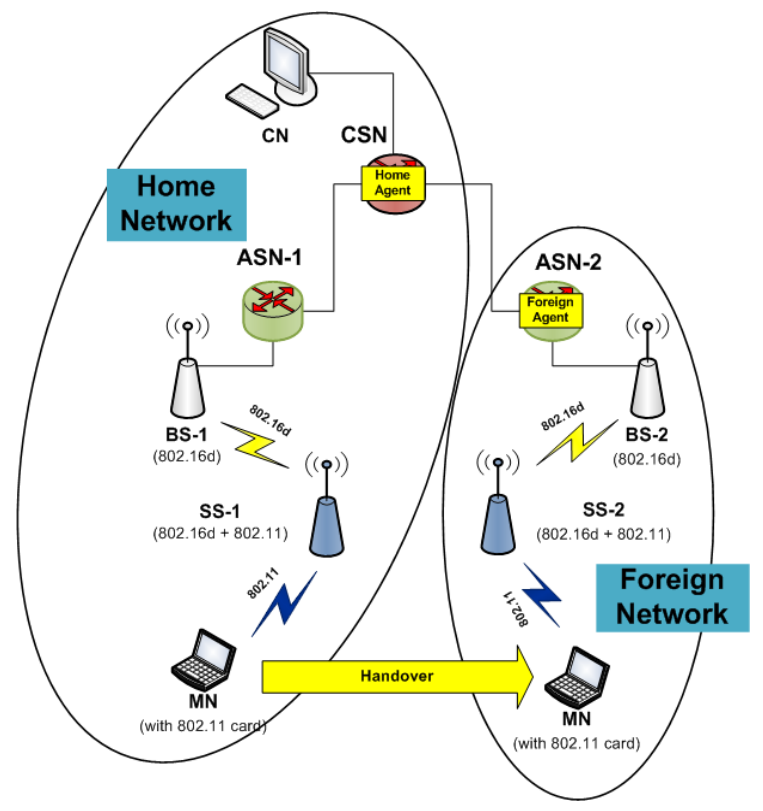

Fig. 4. Schema of the handover procedure

In the triggering phase, the MN detects a new network and reports to the ASN. Then, on the second phase, the resource provision on the next domain is performed, in order to support the "make-before-break" handover process. Finally, on the decision and last phase, the MN moves to the new network. The phases of the handover procedure are described in greater detail in the following subsection. 


\subsubsection{Triggering phase}

Initially, the MN has network connectivity through ASN-1. While connected to this network, the MN will detect a new network controlled by ASN-2 through the information gathered by the Low Level Agent (LLA). When the LLA detects that the actual connection is losing quality and perceives a better one, it sends a trigger to MIHF. Then, the MIHF will generate a "MIH_Link_Going_Down" event, which is sent towards the Mobility Manager (MM) of ASN-1, through the MIH-NSLP protocol.

Upon receiving the event notification, the MM of ASN-1 needs to contact the ASN responsible for the newly detected network, ASN-2. There are two ways to perform this communication, one through the Reference Point R4 and the other through Reference Point R3. In this scenario, ASNs are controlled by different entities and therefore the communication between ASN-1 and ASN-2 is through R3.

\subsubsection{Provisioning}

The resource provisioning in ASN-2 must be preceded by the signalling between ASN-1 and ASN-2. For that purpose, ASN-1 needs to know the IP address of ASN-2. This information is obtained from the MIIS database in the CSN, by sending the "MIH_Get_Information.Request" event, which includes, among other, information about the new network. Upon receiving this request, the CSN will make a query to the MIIS database. If the information is not available or is deprecated, the CSN will redirect the request towards ASN-2. Then, the required information will be encapsulated in a "MIH_Get_Information.Response" message. When receiving this message, the CSN will update the MIIS database and then it will send a response back to ASN-1. At this point, the MM in the ASN-1 has information about ASN-2, and is therefore able to request a new reservation on $\mathrm{ASN}-2$, before prompting the $\mathrm{MN}$ to perform the handover.

The signalling between ASN-1 and ASN-2 is done through a new NSLP, called Mobility Manager NSLP (MM-NSLP). Thus, a MM-NSLP message sent from ASN-1 towards ASN-2 carries the reservation information and the MAC Address of the MN. This last parameter will be useful for the interaction with the DHCP Server.

The MM of ASN-2 starts the local signalling and necessary procedures to guarantee the soft handover. In this way, when the MN moves from the ASN-1 to ASN-2, the resource reservation will be already established. In order to perform the reservation in the Foreign Network, the MM will emulate a request to the DHCP server with the MAC Address of the MN, which was sent in the request made by ASN-1. In fact, the MM will contact the DHCP Manager, entity that was created with the purpose of managing all the interactions with the DHCP server. Afterwards, the DHCP Manager returns the newly assigned IP address, and the MM is able to perform the QoS reservation with the IP address that the $\mathrm{MN}$ will have in the Foreign Network. Then, the MM communicates with the CSC_ASN (a core module responsible to manage the QoS signalling in the WEIRD architecture), which will resort to QoS-NSLP to achieve the signalling needed for QoS Reservation, both in the WiMAX link between BS-2 and SS-2, and between ASN-2 and CSN. In case of 
success, the MM of ASN-2 will inform its homologous of ASN-1 accordingly, using the MM-NSLP. If the reservation fails, an error message is sent instead.

\subsubsection{Decision}

Given that the resources are provisioned on the Foreign Network, the MN must be informed to change to the new network. To accomplish this, the ANS-1 MM asks MIHF to send a "MIH_Link_Action.Request" event to the MN. This event will then force the $\mathrm{MN}$ to move to the new network.

When the MN shifts to the new network, it will send a DHCP Request and the normal negotiation of DHCP will be performed. In this case, the MAC Address of the MN already exists in the leases of DHCP, since the registry was already made by the DHCP Manager of ASN-2. Therefore, the IP Address assigned to the MN is the same as the one for which the reservation was made.

After the MN has moved to the Foreign Network, it will start to receive Router Advertisements of the Foreign Agent (FA) present in the ASN-2 network. Upon the reception of these advertisements, MIP in the MN will send a registration request to the FA. This registration will force the creation of the new IP Tunnel between this FA and the Mobile Node Home Agent, which is the CSN. This tunnel, and the respective registration in the CSN, will allow that the messages for the $\mathrm{MN}$ may be re-directed to its FA, and later on delivered to the Mobile Node itself.

\section{$5 \quad$ Evaluation Setup and Results}

This section presents the results of the evaluation, on a test-bed, of the components developed to support the WIMAX "make-before-break" macro-mobility scenario.

\section{$5.1 \quad$ Test conditions}

Fig. 4 depicts the configuration of the test-bed, composed by two subnets, each one with an ASN. In order to evaluate the scalability of the proposed approach, the tests were performed with different simultaneous reservations, which included 1, 2, 4, 8, $16,32,64$ and 128 sets. The reservations in the WiMAX segment (i.e. between the BS and the MS/SS) were simulated using a dummy Resource Control (RC) module. As the performance of the WiMAX equipment was not in the evaluation scope, further details can be found in [14]. Each test included 5 runs, moreover all the results, depicted with greater detail in the next subsections, are based on average values.

\subsection{Results}

The main goal of this study was to evaluate the overhead introduced by the mechanisms developed in the mobility architecture to enable the "make-before-break" approach. Therefore, the performance of the MM module was the main focus of the evaluation. Additionally, the performance of the modules of the WEIRD system involved in signalling, both mobility and QoS, have been assessed. 
Fig. 5 shows the size of the context transfer messages exchanged between ASN-1 and ASN-2, for the different number of simultaneous reservations. Each message contains all the required information for the handover process, such as source address, destination address and direction.

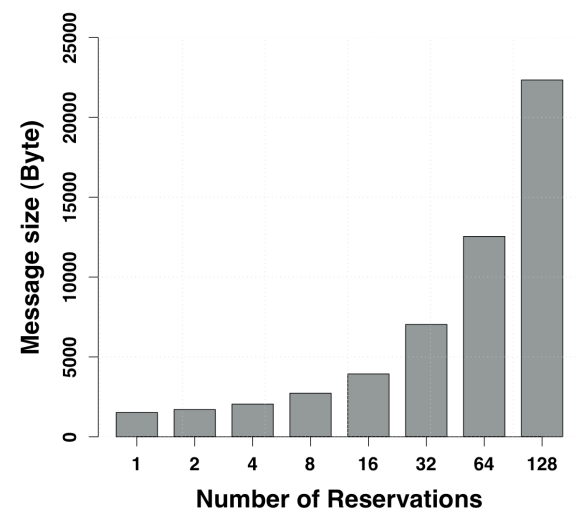

Fig. 5. Context transfer message size

As expected, the message size increases with the number of reservations, due to the QoS specification (QSpec) data, AAA credentials and session identification information associated with each reservation.

The processing time of the Mobility Manager is illustrated in Fig. 6. The graphic shows that the processing overhead introduced by the MM is within acceptable bounds (bellow $80 \mathrm{~ms}$ ) and it is not significantly affected by the number of reservations, for up to 64 simultaneous reservations.

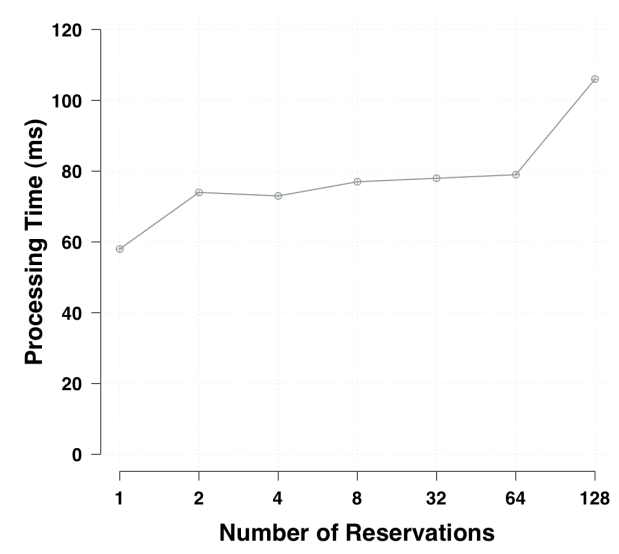

Fig. 6. MM processing time

Fig. 7 depicts the comparison of the required time for resource reservation in the home and foreign networks. It is noticeable that the time required to accomplish 
10 Vitor Bernardo, Luis Cordeiro, Isidro Caramelo, Bruno Sousa, Marilia Curado, Edmundo Monteiro

reservations in both domains is very similar, increasing with the number of reservations. Nevertheless, the reservation time only becomes unbearable for larger number of simultaneous reservations (i.e. above 32 ).

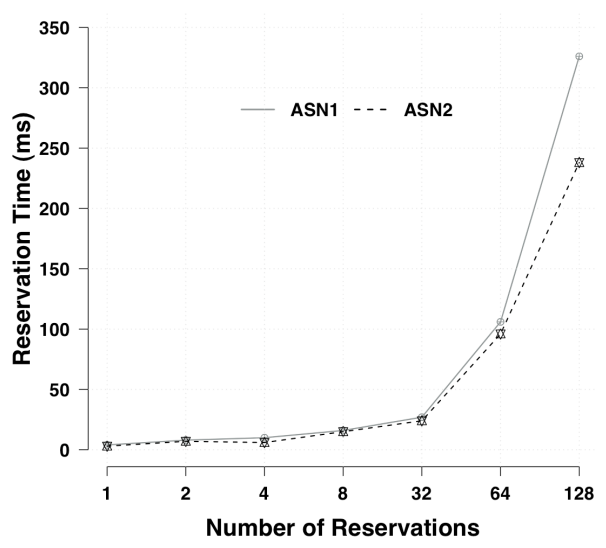

Fig. 7. Resource reservation time in home and foreign networks

Fig. 8 shows the duration of the handover preparation phase, detailing the processing time associated with the main modules involved, in mobility mechanisms, QoS signalling, and AAA functions. The handover preparation time includes the time between the instant when the MM in the ASN-1 receives the information about the bad quality of its link or detection of an available link with higher quality, and the arrival of the response from ASN-2 acknowledging the success of the reservation. The time spent in the CSC, the interaction with AAA, the Resource Management Function (RMF) and the RC processing time are the main modules also evaluated. The length of the handover preparation phase is mainly influenced by the CSC+AAA+RC processing time, due to the complexity of the RMF function of the CSC. Namely, the CSC is responsible for policy and admission control functions, such as QSpec deserialization and communication with the $\mathrm{RC}$ to perform resource reservation in the WiMAX segment, as presented in [14]. 


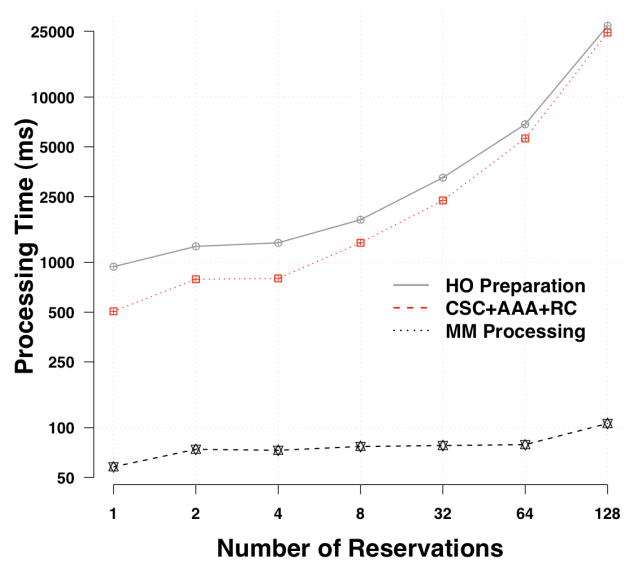

Fig. 8. Handover preparation time

Fig. 8 confirms that the MM processing time is very small (bellow $110 \mathrm{~ms}$ ) even for the highest number of reservations.

In summary, the results show that the modules developed have the potential to achieve a "make-before-break" handover with a limited overhead. Moreover, the CSC proved to be the most critical module of the WEIRD system, both in fixed and mobile scenarios.

\section{Conclusions}

Wireless mobile network access has become, more than a requirement, a fundamental element of nowadays lifestyle. WiMAX has the potential to play an important role in all-IP networks, supporting different levels of service for applications with stringent quality of service requirements, such as voice and video. In this context, the WEIRD project has explored and enhanced the WiMAX capabilities integrated in an end-toend IP architecture. A proposal for macro-mobility following a "make-before-break" approach using the WiMAX technology within the WEIRD project architecture has been specified and evaluated in this paper. In order to achieve the desired objectives, a new NSIS NSLP was developed to support the communication of the MM Modules of different domains. The results of the evaluation of the proposed scheme on a real test-bed showed that the modules designed to support macro mobility introduce a minor impact on the performance of the system, despite the number of simultaneous reservations.

Further work will address the evaluation of IPv6 within the proposed scenario.

Acknowledgments. This work was conducted within the framework of the IST 6th Framework Programme Integrated Project WEIRD (IST-034622), which is partially funded by the Commission of the European Union. The authors acknowledge also all the partners involved in the WEIRD project. 
12 Vitor Bernardo, Luis Cordeiro, Isidro Caramelo, Bruno Sousa, Marilia Curado, Edmundo Monteiro

\section{References}

1. WiMAX Extensions to Isolated Research Data Networks - WEIRD project Description of Work - IST FP6 Integrated Project.

2. E. Guainella, E. Borcoci, M. Katz, P. Neves, M. Curado, F. Andreotti, E. Angori, "WiMAX Extension to Isolated Research Data networks: the WEIRD System", "Mobile WiMAX", Kwang-Cheng Chen (Editor), J. Roberto B. de Marca, Wiley 2008.

3. IEEE 802.16-2004 Standard for Local and Metropolitan Area Networks. Part 16: Air Interface to fixed and Mobile Broadband Wireless Access Systems.

4. IEEE 802.16-2005 Standard for Local and Metropolitan Area Networks Part 16: Air Interface to fixed and Mobile Broadband Wireless Access Systems. IEEE.

5. Broadband Radio Access Networks (BRAN); HiperMAN; Data Link Control (DLC) layer . V1.3.2.

6. Broadband Radio Access Networks (BRAN); HiperMAN; Physical (PHY) layer. V1.3.2

7. WiMAX Forum Network Architecture (Stage 2: Architecture Tenets, Reference Model and Reference Poins). Release 1, version 1.2. WiMAXForum, January 11, 2008

8. IEEE Std 802.21-2008 "IEEE Standard for Local and metropolitan area networks- Part 21: Media Independent Handover", 21 January 2009

9. R. Hancock, G. Karagiannis, J. Loughney, S. Van den Bosch "Next Steps in Signalling (NSIS): Framework", IETF, RFC 4080, June 2005

10. Internet Engineering Task Force Next Steps in Signalling Working Group. http://www.ietf.org/html.charters/nsis-charter.html

11. Schulzrinne, R. Hancock, "GIST: General Internet Signalling Transport", draft-ietf-nsisntlp, IETF, 30 October 2008. GIST

12. J. Manner, G. Karagiannis, A. McDonald, "NSLP for Quality-of-Service Signalling”, draftietf-nsis-qos-nslp, IETF, 7 February 2008.

13. L. Cordeiro, M. Curado, P. Neves, S. Sargento, G. Landi, X. Fu, "Media Independent Handover Network Signalling Layer Protocol (MIH NSLP)", draft-cordeiro-nsis-mih-nslp, IETF, February 18, 2008.

14. B. Sousa, P. Neves, G. Leão, D. Palma, J. Silva, S. Sargento, F. Fontes, M. Curado, F. Boavida, "The Cost of Using IEEE 802.16d Dynamic Channel Configuration", IEEE International Conference on Communications (ICC 2008), Beijing, China, 19-23 May

15.Kumudu .S. Munasinghe and A. Jamalipour, "An architecture for mobility management in interworked 3G cellular and WiMAX Networks," in Wireless Telecommunications Symposium, 2008. WTS 2008, 2008, 291-297..

16. Roland Bless et al., "A quality-of-service signaling architecture for seamless handover support in next generation, IP-based mobile networks," Wirel. Pers. Commun. 43, no. 3 (2007): 817-835..

17.Hui-Juan Yao, Geng-Sheng Kuo, "An Integrated QoS-Aware Mobility Architecture for Seamless Handover in IEEE 802.16e Mobile BWA Networks", Military Communications Conference, 2006. MILCOM 2006, 23-25 Oct. 2006.

18. Ashutosh Dutta et al., "Seamless proactive handover across heterogeneous access networks," Wirel. Pers. Commun. 43, no. 3 (2007): 837-855.

19. Lampropoulos, G. Salkintzis, A.K. Passas, N., "Media-independent handover for seamless service provision in heterogeneous networks", Communications Magazine, IEEE, January 2008 . 